\title{
La sensibilité radioécologique des territoires : vers un outil opérationnel - le projet SENSIB
}

\author{
C. MERCAT-ROMMENS ${ }^{1}$, S. ROUSSEL-DEBET ${ }^{1}$, B. BRIAND ${ }^{1}$, V. DURAND ${ }^{1}$, \\ B. BESSON ${ }^{1}$, P. RENAUD $^{1}$
}

(Manuscrit reçu le 5 décembre 2006, accepté le 15 février 2007)

RÉSUMÉ La sensibilité radioécologique représente l'intensité de la réponse globale d'un territoire à une pollution radioactive chronique ou accidentelle. Les perspectives d'application opérationnelle de ce concept sont explorées par l'IRSN dans le cadre du projet SENSIB. Le premier enjeu de SENSIB consiste à représenter globalement les conséquences territoriales d'une situation de contamination radioactive, ce qui nécessite de rassembler et d'analyser les données radioécologiques et contextuelles. À cet effet, diverses méthodes innovantes de traitement de la connaissance sont explorées. Le deuxième enjeu est de concevoir un outil unique de gestion de cette connaissance partagé par différents intervenants (autorités, population, experts), ce qui est envisagé par l'application de méthodes d'aide à la décision. La stratégie, les résultats préliminaires et les axes de recherche du projet SENSIB sont brièvement présentés.

ABSTRACT The radioecological sensivity of territories: towards an operational tool through the SENSIB Project.

Radioecological sensitivity represents the intensity of a region's global reaction to an accidental or chronic radioactive pollution. The prospects for an operational application of this concept are investigated by IRSN within the framework of the SENSIB project. The first aim of SENSIB is to represent globally the territorial consequences of a situation of radioactive contamination, which requires to collect and to investigate the radioecological and contextual data. For this purpose, varied innovative methods of knowledge treatment are investigated. The second objective is to elaborate a management tool of this knowledge which could be shared by various stakeholders (authorities, population, experts), and that is envisaged by the application of supporting methods to decision-making. The strategy, the preliminary results and the avenues of research of the project SENSIB are briefly presented.

Keywords: environmental pollution / radionuclides / vulnerability / sensitivity factor / methods / operational tool

1 IRSN, Laboratoire d'Étude Radioécologique en milieux Continental et Marin, DEI/SESURE/LERCM, Cadarache, bâtiment 153, B.P. 3, 13115 St-Paul-lez-Durance Cedex, France. 


\section{Introduction}

Au cours de la dernière décennie, les attentes vis-à-vis de la radioécologie et plus généralement de l'évaluation des risques associés aux installations nucléaires ont considérablement évolué. Le «calcul de dose » pour un individu de référence, ou un groupe de référence, exposé aux rejets chroniques ou accidentels de radioactivité dans l'environnement et la comparaison directe des doses issues de ce calcul avec les seuils réglementaires ne suffisent plus. Cela est à la fois vrai d'un point de vue scientifique, puisque l'analyse des incertitudes associées aux calculs de doses devient la norme, mais aussi d'un point de vue sociétal car la population ne se reconnaît pas dans les hypothèses simplificatrices des scénarios d'expositions. L'objectif du projet SENSIB, «sensibilité radioécologique », engagé en 2003 par l'IRSN, est de contribuer à l'évolution des outils, méthodes et savoirs de la radioécologie pour répondre aux nouvelles attentes de la société. Le projet SENSIB porte principalement sur l'acquisition de connaissances relatives aux spécificités locales des territoires et notamment sur celles — appelées dans ce cadre «facteurs de sensibilité » — qui vont s'avérer prépondérantes dans l'évaluation des risques. Le projet SENSIB vise aussi à développer les méthodes de traitement mathématique nécessaires pour prendre en compte cette évolution de la connaissance. Enfin, dans le cadre du projet, il est prévu de vérifier que les évolutions proposées correspondent aux attentes sociétales des acteurs, qu'ils soient des responsables de la décision ou des représentants de la population.

Le projet SENSIB est actuellement orienté principalement sur l'étude des milieux agricoles du territoire métropolitain français en contexte post-accidentel. L'objectif de cet article est de présenter la méthodologie, les principaux enjeux, les premiers résultats concluants et les perspectives de ce programme de recherche.

\section{Le projet SENSIB}

Le projet SENSIB est basé sur le concept de la sensibilité radioécologique qui est destiné à représenter l'intensité de la réponse globale de l'environnement à une pollution. Le concept de sensibilité radioécologique est apparu pour la première fois en 1979 dans le cadre de l'étude des conséquences des retombées des essais nucléaires atmosphériques (Aarkrog, 1979). La sensibilité radioécologique était définie comme « la concentration d'un radionucléide dans un échantillon de l'environnement résultant d'un dépôt de $1 \mathrm{mCi} \mathrm{km}^{-2}$ de ce radionucléide ». L'indicateur de mesure de la sensibilité radioécologique utilisé par Aarkrog était alors un facteur de transfert agrégé (Tag) exprimé en $\mathrm{Ci} \mathrm{kg}^{-1}$ par $\mathrm{mCi} \mathrm{km}{ }^{-2}$. En 1999, le concept de vulnérabilité fut utilisé dans le cadre du projet AMAP - Arctic Monitoring and Assessment Programme - (Strand et al., 1997). Le groupe de 
travail montrait notamment que les écosystèmes arctiques étaient plus vulnérables à la contamination au radiocésium que les écosystèmes des climats tempérés et identifiait les facteurs à l'origine de cette vulnérabilité. Le travail sur la vulnérabilité des écosystèmes arctiques a ensuite été poursuivi jusqu'en 2002 dans le cadre du programme AVAIL - Arctic Vulnerability to radioactive Contamination - (AVAIL, 2002). Le concept de « critical loads » (charge critique), développé initialement pour représenter l'impact des émissions acides, fut aussi proposé comme indicateur de la sensibilité radioécologique. La charge critique est définie comme « l'estimation du niveau d'exposition à un ou plusieurs polluants en dessous duquel, dans l'état actuel des connaissances, aucun effet délétère significatif sur l'environnement n'est susceptible de survenir $»$. Le concept de charge critique a été largement utilisé par la suite : en Hongrie pour évaluer le transfert du césium-137 dans les produits alimentaires après un dépôt accidentel, en Suède pour comparer le comportement de différents radionucléides dans le sol dans différentes régions agricoles (Eriksson, 1997) et dans le cadre des projets SAVE - Spatial Analysis of Vulnerable Ecosystems - (Howard et al., 1999) et ARMARA (Iosjpe et al., 2002).

La réflexion théorique la plus approfondie sur le thème de la sensibilité radioécologique est celle réalisée dans le cadre du Forum « Radioecological Sensitivity » qui a eu lieu entre septembre 1998 et mars 2001 (Howard et al., 2002). Ce forum, animé par le Center for Ecology and Hydrology, a rassemblé des représentants de différents instituts et universités afin de définir la sensibilité radioécologique, de l'illustrer par des exemples d'applications et de proposer des recommandations pour les utilisateurs de ce concept.

Le projet SENSIB a pour objectif d'explorer la dimension opérationnelle de ce concept en explicitant les relations qui lient les facteurs de sensibilité à des indices de la sensibilité des territoires et en construisant des outils qui permettent de représenter et de hiérarchiser ces indices (Mercat-Rommens et Renaud, 2005a, 2005b). En effet, les conséquences pour l'homme et l'environnement d'une pollution radioactive dépendent de l'importance et de la nature de celle-ci, mais aussi de l'environnement qui la reçoit. Qu'elles s'expriment en termes de risques sanitaires, de conséquences économiques, ou de préoccupations écologiques, ces conséquences seront plus ou moins pénalisantes suivant les caractéristiques du milieu touché et l'usage qu'en fait l'homme. Ainsi, les différents milieux, urbains, agricoles, forestiers, fluviaux, lacustres, marins ou d'altitude, présentent des sensibilités différentes vis-à-vis d'une pollution. Par exemple, la sensibilité des milieux urbains est liée à la densité de population et aux surfaces artificielles susceptibles de concentrer les polluants. Celle des espaces agricoles provient de l'atteinte de la chaîne alimentaire par la contamination du sol, des plantes cultivées et des animaux d'élevage. Les espaces naturels, notamment les espaces forestiers, 


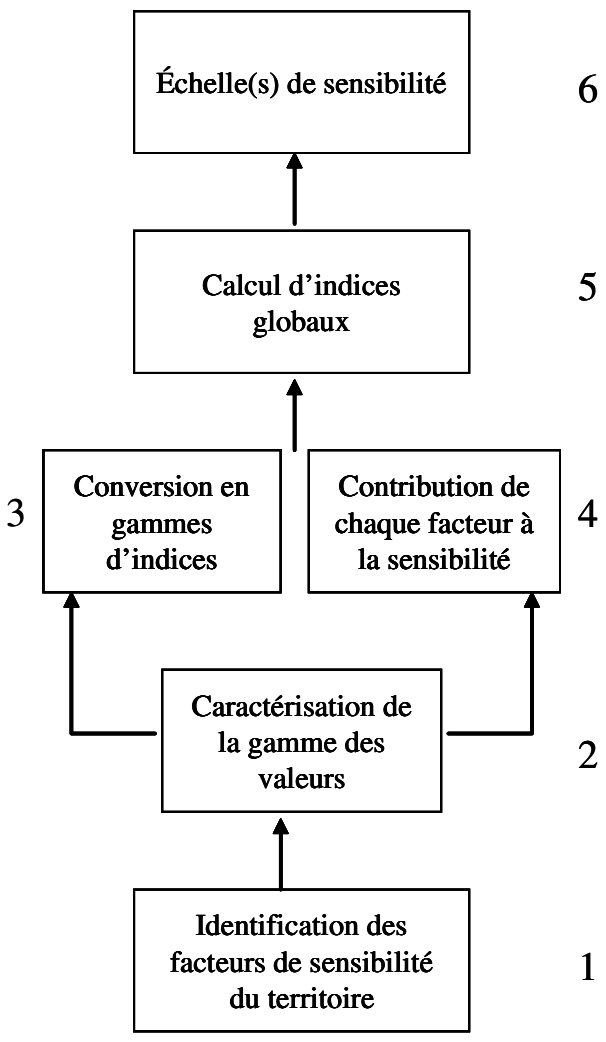

Figure 1 - Méthodologie générale du projet SENSIB.

General methodology of the SENSIB project.

sont connus pour entretenir la rémanence des polluants qui y sont en permanence recyclés ou transformés. Ce sont souvent dans ces milieux que les espèces végétales et animales présentent les concentrations les plus élevées en radionucléides. Au sein même de chacune des grandes composantes environnementales, différents facteurs, naturels ou anthropiques, spécifiques à l'écosystème considéré, déterminent la réponse de l'environnement à une pollution.

\section{Méthodes}

Le projet SENSIB est structuré en cinq étapes résumées sur la figure 1 :

(1) l'identification des facteurs de sensibilité consiste à déterminer, pour un scénario de contamination donné, quels paramètres vont influencer de façon prépondérante la réponse du compartiment environnemental étudié ; 
(2) la caractérisation des gammes de valeurs des facteurs de sensibilité : il s'agit de connaître au mieux la distribution de valeurs de chacun des facteurs précédemment identifiés à l'échelle du territoire métropolitain français, en fonction de caractéristiques qui peuvent être spatialisables (par exemple, une donnée météorologique) ou typologiques (par exemple un comportement de la population) ;

(3) la conversion des gammes de valeurs en gammes d'indices, afin d'aboutir à une normalisation de données disparates ;

(4) l'évaluation du poids de chaque facteur de sensibilité sur la sensibilité globale à partir des informations spécifiquement liées au territoire considéré ;

(5) le calcul d'indices globaux, en vue de définir un outil opérationnel : l'échelle de sensibilité radioécologique d'un territoire.

La réalisation de ces étapes nécessite de rassembler les données radioécologiques (mesures de terrain, résultats expérimentaux, modélisation) et contextuelles (écosystèmes et modes de vie), puis de les traiter par une approche commune. À cet effet, est étudiée l'utilisation d'outils mathématiques innovants (théorie de Dempster-Shafer, test géostatistique de Moran, méthode CART) dont certains sont encore en cours de développement. Enfin, la production d'un outil opérationnel et la mise à disposition de cet outil auprès des parties prenantes sont envisagées en recourrant à des outils d'aide à la décision (méthodes d'analyse multicritères).

\section{Identification et caractérisation des facteurs de sensibilité}

L'exécution de la phase d'identification et de caractérisation des facteurs de sensibilité (étapes 1 et 2, Fig. 1) passe par l'utilisation de modèles de transfert des radionucléides dans l'environnement dont on sélectionne les équations nécessaires à l'évaluation des transferts dans les compartiments de l'écosystème étudiés. Les premiers travaux de SENSIB portent sur l'évaluation des transferts aux productions agricoles via le compartiment atmosphérique de quelques radionucléides choisis en tant qu'exemples $(\mathrm{Cs}, \mathrm{Sr}, \mathrm{I})$. Les équations utilisées sont celles qu'utilise classiquement la radioécologie pour calculer les concentrations massiques des radionucléides dans les productions végétales et animales soumises à un dépôt; selon les scénarios envisagés, elles sont issues, des codes opérationnels tels que FOCON (Rommens et al., 1999), ECOSYS (Müller et Pröhl, 1993) ou ASTRAL (Mourlon et Calmon, 2002). Les paramètres étudiés sont ceux dont on présume qu'ils peuvent être des facteurs de sensibilité, de par leur variabilité spatiale et/ou typologique et la sensibilité statistique des équations aux valeurs que prennent ces paramètres. L'examen des données radioécologiques et contextuelles est alors possible par deux approches. L'une d'elle consiste à analyser des séries de mesures de radioactivité effectuées dans l'environnement et 
à rechercher les corrélations entre diverses caractéristiques de la production considérée (par exemple le rendement ou la date de floraison d'une culture qui interviendra sur l'activité massique à la récolte) et les valeurs réellement mesurées. La seconde consiste à établir directement la distribution (gamme de valeurs et variabilité) des paramètres des équations et à déterminer leur importance relative afin d'en déduire un nombre réduit de facteurs de sensibilité. Ces deux approches sont illustrées ci-après.

\subsection{Confrontation mesures in situ - valeurs des paramètres des modèles}

Dans une étape préliminaire du projet, des séries de mesures in situ de végétaux ont été confrontées aux estimations d'un modèle classique de transfert aux végétaux par voie racinaire et foliaire. L'objectif était d'obtenir les distributions statistiques, si possible régionalisées, des paramètres de l'équation de transfert des radionucléides vers les végétaux et de comparer le modèle aux mesures. Cette approche s'est fondée sur l'existence de séries de résultats de mesures de ${ }^{90} \mathrm{Sr}$,

${ }^{137} \mathrm{Cs}$ et divers radionucléides à vie courte dans des légumes-feuilles acquises en France sur la période 1960-1980 durant les retombées des essais atmosphériques d'armes nucléaires (Renaud et Louvat, 2004). Les différents cas étudiés ont cependant montré une mauvaise qualité de l'ajustement entre le modèle et les mesures (Fig. 2), et ce malgré l'importance inhabituelle (plus de 4000 résultats de mesures) des jeux de données disponibles pour l'étude (Briand et MercatRommens, 2006 ; Briand et al., 2006). Cet exercice a notamment démontré de façon rigoureuse que, pour pouvoir utiliser des mesures in situ afin de renseigner un modèle de transfert, le nombre de mesures, souvent invoqué comme principal facteur limitant à leur interprétation, n'est qu'un aspect de la possibilité de leur exploitation ultérieure et que la qualité du renseignement qui accompagne l'acquisition de ces mesures est un élément fondamental pour pouvoir les interpréter. Dans cet exemple, il s'est avéré qu'il était indispensable de connaître le plus précisément possible le terme source (ici l'activité déposée) et les caractéristiques des prélèvements; ce travail a ainsi permis de proposer des améliorations pour l'acquisition de données futures, mais s'est révélé infructueux pour l'objectif du projet.

\subsection{Actualisation et structuration des données agronomiques régionalisées}

Une approche différente a alors été engagée ; du fait de l'importance primordiale des caractéristiques territoriales (climat, type de sol, type de pratiques agricoles) sur la sensibilité d'un territoire, l'acquisition de données agricoles récentes et leur analyse ont été réalisées afin d'identifier, pour chaque production agricole, les 




Figure 2 - Exemple de résultats de la confrontation des mesures in situ aux valeurs des paramètres des modèles sur le cas du poireau issu de (Briand et Mercat-Rommens, 2006).

Example of comparison between environmental measurements and modelling parameters the leek case from (Briand and Mercat-Rommens, 2006).

données les plus influentes sur la sensibilité. Cette approche accomplie pour les productions céréalières (Mercat-Rommens et al., 2006b), les prairies permanentes (Durand et al., 2007), et en cours sur la vigne, la filière prairies $\rightarrow$ lait $\rightarrow$ fromage et les légumes-feuilles a notamment permis de montrer toute la richesse du couplage entre le code radioécologique ASTRAL et un modèle agronomique de culture comme STICS (Brisson et al., 2003). STICS (Simulateur mulTIdisciplinaire pour les Cultures Standard) est un modèle de fonctionnement des cultures développé par l'INRA depuis 1996. Son principal objectif est de simuler les conséquences des variations du milieu et du système de culture sur la production d'une parcelle agricole. À partir de la caractérisation du climat, du sol, de l'espèce cultivée et des techniques culturales appliquées, le modèle STICS calcule des variables de sortie relatives à la production. Ce couplage permet, pour certains paramètres radioécologiques ou contextuels, de remplacer les valeurs génériques utilisées par défaut dans le modèle ASTRAL par des valeurs adaptées aux caractéristiques agricoles régionales calculées par le modèle STICS (Fig. 3). Cette prise en compte des données récentes et réalistes concernant les pratiques culturales en France est un axe de travail important car, d'une part, il est évident qu'une amélioration de la 


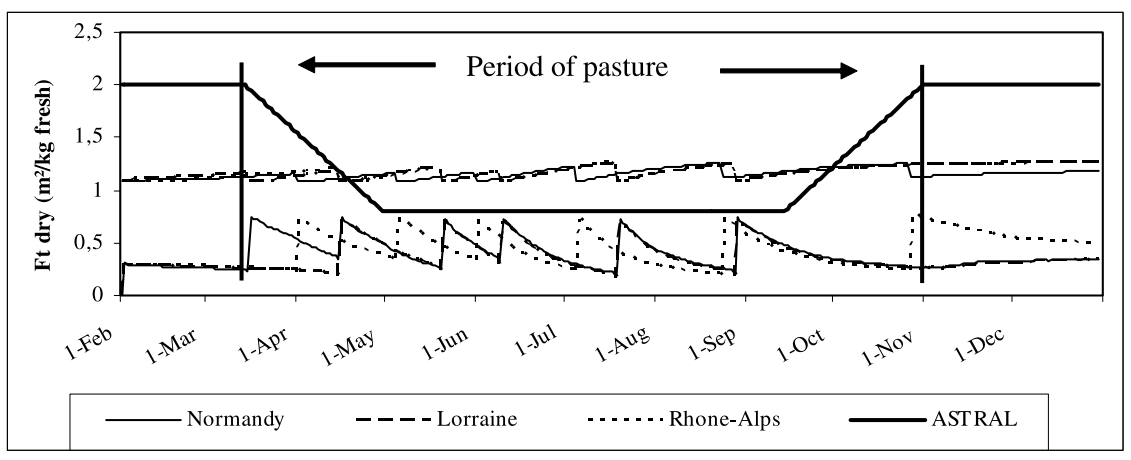

Figure 3 - Comparaison des valeurs de transfert direct du dépôt à l'herbe de pâture $\left(F_{\text {dry }}\right)$ du modèle radioécologique ASTRAL aux données régionalisées issues du modèle agronomique STICS (Durand et al., 2007).

Comparison of the value of direct transfer from deposit to permanent pasture $\left(F_{\text {dry }}\right)$ according to radioecological model ASTRAL and the regional data resulting from the agronomical model STICS from (Durand et al., 2007).

prédiction post-accidentelle est fondamentale pour diminuer les incertitudes paramétriques, et d'autre part, l'amélioration des pratiques de gestion des conséquences d'une pollution radioactive repose en partie sur la crédibilité du dialogue technique entre les experts institutionnels et les professionnels du monde agricole et agro-alimentaire. La bonne connaissance des pratiques est un élément indispensable de ce dialogue et une réflexion stratégique sur les moyens opérationnels à mettre en place pour acquérir et mettre à jour régulièrement ce type de données est une des finalités ultérieure du projet, en liaison avec le programme COPT (Conception d'Observatoires de Pratiques Territorialisées) de l'INRA (Le Ber et al., 2006).

\subsection{Acquisition de données d'exposition de la population}

Outre les données d'occupation du sol, la sensibilité d'un territoire est aussi déterminée par l'ampleur de l'exposition humaine qui relève principalement du potentiel d'ingestion par l'homme des aliments produits localement. Actuellement, les données disponibles sur les taux d'autoconsommation des populations françaises proviennent de statistiques nationales (INSEE, 1991). Une des actions du projet SENSIB est d'améliorer la connaissance des comportements alimentaires des populations potentiellement sensibles à l'échelle des territoires. À titre exploratoire, une enquête alimentaire a été réalisée en 2004-2005, en collaboration avec AREVA et le Bureau d'études de gestion et d'aménagement du territoire (BEGEAT-Toulon), à proximité du site nucléaire de PierrelatteTricastin. Cette étude a notamment montré que les taux d'autoconsommation de 
certains groupes de population rurale pouvaient encore actuellement dépasser les $90 \%$ pour certaines catégories de légumes (Durand et al., 2006). Les résultats de l'étude de Pierrelatte-Tricastin doivent maintenant être comparés à ceux d'autres études réalisées précédemment autour de sites nucléaires (Descamps et Guillet, 2003) pour estimer la fréquence d'occurrence de ce type de comportements particuliers. Ces résultats laissent néanmoins présager l'importance de la connaissance de certaines habitudes de vie spécifiques des territoires, et renforcent les enjeux associés à la création d'observatoire des pratiques comportementales locales. Le projet SENSIB contribuera à ces enjeux par une revue méthodologique des procédures d'enquêtes, revue basée sur le retour d'expérience des enquêtes auprès des populations riveraines des installations nucléaires.

\section{Mise en ouvre de méthodes de traitement de la connaissance}

Les données concernant les territoires, qu'elles soient radioécologiques ou non, forment le matériau de base du projet mais le défi technique de SENSIB est de développer des méthodes innovantes permettant d'organiser ces données en leur donnant un sens opérationnel (étapes 3 à 5, Fig. 1). Trois sujets particulièrement critiques lors du traitement des données sont actuellement étudiés : la représentation et la prise en compte des incertitudes, le traitement de la variabilité et des diversités d'échelles spatiales voire temporelles, la hiérarchisation et l'agrégation de données.

\subsection{Le traitement de l'incertitude}

En évaluation des risques, l'analyse d'incertitude est devenue une étape incontournable de la restitution des résultats, en termes de rigueur scientifique et de crédibilité sociétale. Cependant, sa réalisation soulève généralement de nombreuses difficultés car la connaissance scientifique dans ces domaines est souvent limitée. L'incomplétude et l'imprécision de la connaissance nécessitent alors fréquemment le recours à une connaissance plus subjective appelée le jugement d'expert. Dans ce contexte, les techniques classiques ne sont pas toujours suffisantes pour rendre compte de la connaissance disponible (Mercat-Rommens et al., 2002). En effet, l'incertitude a surtout été appréhendée dans un cadre purement probabiliste, c'est-à-dire que l'information sur les paramètres des modèles est supposée de nature aléatoire (variabilité). Cette approche consiste à représenter les paramètres incertains par des distributions de probabilités uniques et à propager l'incertitude relative à ces paramètres vers la valeur du risque encouru par la cible, en appliquant en général les techniques dites de Monte Carlo (nombreux tirages aléatoires dans les distributions de chaque paramètre pour 
déduire une distribution du risque). Si cette approche est bien connue, toute la difficulté tient à une définition cohérente, par rapport à la connaissance disponible, des distributions de probabilités affectées aux paramètres. En effet, lorsque l'information dont on dispose est imprécise, le calage d'une distribution de probabilité unique sur ce type de connaissance devient subjectif et en partie arbitraire. De plus, dans le processus de propagation, la connaissance (ou méconnaissance) des dépendances et/ou des corrélations entre les paramètres peut avoir des répercussions importantes sur le résultat en fonction des hypothèses que l'on peut faire ; lorsque le traitement des incertitudes est appréhendé dans un cadre purement probabiliste, le fait de supposer l'indépendance entre les paramètres a pour effet de créer des phénomènes de compensation des erreurs. Cela se ressent surtout sur les «queues de distributions », alors même que c'est justement à ces scénarios extrêmes que s'intéresse l'évaluateur de risque.

Dans le cadre de SENSIB, un des premiers objectifs du travail réalisé par Baudrit (2005) a été de promouvoir la cohérence entre la manière dont on représente l'information dans les modèles de risque et l'information dont on dispose réellement. Ainsi, les deux sources d'incertitudes, stochastique (aléatoire) et épistémique (imprécision) doivent-elles être traitées de manière différente : l'information aléatoire est traitée de manière rigoureuse par les distributions de probabilités classiques, alors que l'information de nature imprécise est mieux traitée par des familles de distributions de probabilités codées par des paires de probabilités cumulées hautes et basses (p-boxes) ou par des distributions de possibilités (aussi appelées «intervalles flous») ou encore par des intervalles aléatoires utilisant les fonctions de croyance de Dempster-Shafer (Dempster, 1967) (Tab. I).

\section{TABLEAU I}

Proposition de typologie entre la nature de la connaissance et l'outil mathématique à utiliser pour la représenter (d'après Baudrit, 2005).

Typology proposed to link nature of knowledge and mathematical tool to represent it (adapted from Baudrit, 2005).

\begin{tabular}{lll}
\hline \multicolumn{1}{c}{ Type de connaissance } & \multicolumn{1}{c}{ Exemple } & Modèle mathématique \\
\hline $\begin{array}{l}\text { Échantillon de taille conséquente } \\
\text { Connaissance théorique des phénomènes } \\
\text { associés }\end{array}$ & $\begin{array}{l}\text { Pluviométrie sur une station de } \\
\text { Météo-France }\end{array}$ & Distribution de probabilités \\
$\begin{array}{l}\text { Échantillon de petite taille } \\
\text { Modèle paramétrique imprécis }\end{array}$ & Facteur de transfert foliaire & p-boxes \\
Avis d'expert & $\begin{array}{l}\text { Gamme de valeurs et/ou valeurs } \\
\text { moyennes attendues pour le pH des } \\
\text { sols agricoles d'une commune }\end{array}$ & Distribution de possibilités \\
$\begin{array}{l}\text { Toute information de nature aléatoire } \\
\text { et imprécise }\end{array}$ & $\begin{array}{l}\text { Résultats d'un sondage exprimés } \\
\text { sous forme de quantiles }\end{array}$ & Fonction de croyance \\
\hline
\end{tabular}


Une fois que la connaissance a été représentée de façon cohérente par rapport à sa nature propre, épistémique ou stochastique, différentes méthodes de propagation des incertitudes à travers les modèles de risque peuvent être développées et sont utilisables en fonction des structures de dépendance entre les paramètres (Baudrit, 2005). Les résultats issus de ces méthodes de propagation des incertitudes sont ensuite utilisables pour en tirer les informations pour une phase de décision et/ou pour orienter les programmes de recherche visant à acquérir de la connaissance supplémentaire. Ces méthodes alternatives ont été implémentées dans des logiciels comme le code SUNSET@ de l'IRSN (Chojnacki et Ounsy, 1996), testées sur des cas réels et comparées à un traitement purement probabiliste, afin de mettre en évidence l'influence de la représentation mathématique de la connaissance et l'influence de la prise en compte des structures de dépendance sur les résultats du calcul de risque (Mercat-Rommens et al., 2005, 2006a). Le travail sur ces théories doit cependant être poursuivi pour développer les moyens d'une utilisation plus aisée et le recours à d'autres fonctionnalités comme l'analyse de sensibilité floue, les calculs inverses. De plus, un effort important est envisagé pour faciliter l'utilisation de ces outils dans le cadre de la prise de décision.

\subsection{Traitement de la variabilité spatiale}

Le traitement de la variabilité spatiale réalisé dans le cadre de SENSIB a notamment pour objectif d'agréger de façon cohérente des données concernant la sensibilité radioécologique d'une zone de production agricole. La démarche générale consiste à identifier, sur la base de simulations numériques, les paramètres régionalisables les plus influents sur la contamination d'une culture pour un scénario donné, à affecter des valeurs régionales à ces paramètres et à mettre en œuvre une analyse géostatistique. Cette analyse géostatistique doit permettre de cartographier les agrégats géographiques de données homogènes (dits «clusters ») et in fine de proposer des regroupements pour visualiser les zones potentiellement les plus sensibles.

Une étude exploratoire a été menée dans ce cadre sur la sensibilité régionale de la culture de blé d'hiver. L'objectif principal était d'établir si un dépôt radioactif accidentel, uniforme à l'échelle de la France, induirait une contamination des céréales identiques sur tout le territoire et sinon, quelle quantité de céréales serait contaminée et où en France, en fonction de la date de l'accident. Trois sources de variabilité ont été étudiées : le climat, le type de sol et les pratiques agricoles (fertilisation, irrigation, calendrier cultural). L'étude s'est appuyée sur les équations du modèle radioécologique ASTRAL de l'IRSN, et le facteur de transfert de la radioactivité (césium-137) de l'air au grain de blé a été plus particulièrement examiné car il dépend de deux paramètres radioécologiques 
régionalisables : la captation (capacité d'interception des radionucléides par les feuilles) et la translocation (potentiel de transfert des radionucléides depuis les feuilles vers les organes consommés). Le modèle agronomique STICS a permis de déterminer la variabilité spatiale du développement foliaire du blé d'hiver, ainsi que les dates d'occurrence des stades végétatifs et notamment de la floraison, variables auxquelles on a pu corréler les paramètres radioécologiques de captation et de translocation. Les sorties des simulations effectuées sur 12 stations climatiques réparties sur la France et sur deux variétés, l'une précoce et l'autre tardive, ont permis d'identifier les facteurs de sensibilité régionaux qui contribuent le plus à la sensibilité radioécologique du blé d'hiver. La date de floraison et le rendement cultural sont apparus comme des facteurs-clés de la sensibilité de cette culture. En effet, la floraison détermine le début d'un transfert préférentiel des contaminants radioactifs vers les grains. Ce paramètre, la date de floraison, peut être tout particulièrement utile pour l'aide à la décision car c'est un stade de la croissance du végétal assez facile à identifier sur le terrain et parce que cette date peut être estimée de façon anticipée par la connaissance de données régionales sur le climat et la variété cultivée. L'indicateur « date de floraison » permet de savoir où, en France, le blé d'hiver serait contaminé tandis que l'indicateur « rendement cultural» indique quelle quantité de céréales serait contaminée. Après généralisation de l'étude sur le blé d'hiver aux principales autres céréales françaises, nous avons déterminé, grâce à l'analyse géostatistique des clusters spatiaux de la variable « rendement cultural », les zones de productions plus ou moins intensives de céréales (Mercat-Rommens et al., 2006b). L'indicateur géostatistique utilisé est le test de Moran (Anselin, 1995 ; Moran, 1950). Cette analyse géostatistique a notamment permis de réaliser une carte de la vulnérabilité des céréales à une contamination radioactive qui fait apparaître clairement les tendances régionales (Fig. 4).

Ce travail sur la prise en compte de la variabilité spatiale va se poursuivre dans le cadre de la contamination d'une filière agricole (prairies $\rightarrow$ lait $\rightarrow$ fromage) pour laquelle va être étudiée la représentativité des mesures acquises sur le terrain pour estimer les indicateurs de contamination de l'environnement, afin de déterminer la stratégie d'échantillonnage la plus pertinente en fonction de la taille des bassins de collecte du lait et de production fromagère.

\subsection{Hiérarchisation des facteurs}

Pour évaluer la contribution des différents facteurs de sensibilité à la sensibilité globale du milieu et proposer un système d'indexation destiné à hiérarchiser ces facteurs, la méthode CART, Classification And Regression Trees (Breiman et al., 1984), est explorée. Cette méthode permet, entre autres, de déterminer les 




Figure 4 - Résultats de l'analyse des clusters locaux pour le cas du rendement global en céréales. Les clusters positifs sont présentés en gris foncé, les clusters négatifs en gris clair.

Results from the analysis of local cluster related to global yield for cereals. The positive clusters are in dark grey and the negative ones in light grey.

combinaisons de plage de valeurs des variables radioécologiques et agronomiques («entrées du modèle radioécologique») qui conduisent à des catégories prédéfinies de l'activité massique d'un aliment («sortie du modèle radioécologique ») par l'établissement d'arbres de classification. L'enjeu de tels outils est particulièrement important en contexte post-accidentel pour la connaissance des associations de variables qui vont conduire à dépasser des seuils prédéfinis. Une première application a été réalisée dans le cas particulier d'une culture de légumes-feuilles (laitue) soumise à un apport atmosphérique de strontium-90, afin d'identifier les caractéristiques des végétaux pour lesquelles cet apport pourrait conduire à dépasser différentes plages de niveaux maximum admissibles dans les denrées alimentaires. Les équations classiques de modélisation du transfert du ${ }^{90} \mathrm{Sr}$ depuis l'atmosphère jusqu'à la salade adaptées du modèle ASTRAL ont été utilisées et 10000 simulations ont été faites, en utilisant pour les paramètres de l'équation des distributions de probabilité de leurs valeurs. L'illustration de cette étude est présentée de façon simplifiée sur la figure 5. Les catégories 1 et 2 de valeurs d'activités massiques issues de l'arbre de classification représentent la contamination de la laitue à la récolte, respectivement 




Figure 5 - Illustration de l'application de la méthode CART : transfert du ${ }^{90} \mathrm{Sr}$ depuis l'atmosphère jusqu'à la salade.

Example of application of CART procedure: transfer of ${ }^{90} \mathrm{Sr}$ from air to salad.

inférieure ou égale et supérieure à $100 \mathrm{~Bq} \mathrm{~kg}^{-1}$ frais. Les associations de plages de valeurs des variables influentes (ici le rapport de captation, le délai dépôt-récolte et la densité de dépôt) conduisant l'activité massique résultante à appartenir aux catégories 1 et 2 sont représentées sur la figure 5, la densité de dépôt étant tirée au hasard entre 20 et $100000 \mathrm{~Bq} \mathrm{~m}^{-2}$. Cette méthode permet ainsi de déterminer quelles sont les variables qui discriminent le mieux l'activité massique par le calcul des variables et des combinaisons de valeurs des variables qui conduisent à de faibles ou fortes valeurs d'activité dans les productions. Ce type de méthodes peut également être utile pour l'aide à la décision.

Ces méthodes vont être appliquées à d'autres légumes-feuilles (poireau, chou, ...) puis on étudiera leur généralisation aux différentes catégories de légumes «ensemble des légumes-feuilles », « ensemble des légumes-racines », « ensemble des légumes-fruits ».

\section{L'aide à la décision}

L'évaluation de la sensibilité radioécologique du territoire français va inévitablement conduire aux calculs de différents indicateurs de la sensibilité : par 
exemple, des activités massiques dans les productions agricoles $\left(\mathrm{Bq} \mathrm{kg}^{-1}\right)$ mais aussi des densités d'activité de dépôt au sol $\left(\mathrm{Bq} \mathrm{m}^{-2}\right)$ et des activités totales contenues dans une récolte à partir d'une surface donnée (Bq). Il faudra donc hiérarchiser ces indicateurs et trouver des moyens de les comparer entre eux. Dans le cadre du projet SENSIB, il est proposé d'explorer les méthodes d'analyses multicritères de type ELECTRE (Maystre et al., 1994) pour permettre des comparaisons et des tris des indicateurs de sensibilité radioécologique. En outre, ces méthodes d'analyse multicritères permettent de prendre en compte les préférences des différents acteurs de la gestion des territoires (décideurs, experts, représentants locaux, ...) même lorsqu'elles sont conflictuelles et peu structurées. Le modèle d'aide à la décision, ultime étape du projet SENSIB (Fig. 6), est alors élaboré en s'appuyant sur ce qui semble être la partie consensuelle de la perception du problème qu'en ont les acteurs. Une méthode d'analyse multicritères va donc être développée dans le cadre du projet SENSIB en cherchant à tirer parti du recensement des facteurs de sensibilité territoriaux (environnementaux et anthropiques) élaboré avec différents acteurs d'un territoire et de leur examen en commun. Le recours à cette méthode d'analyse multicritères permettra notamment de modéliser et de formaliser la préparation de la décision dans le contexte postaccidentel.

\section{Perspectives d'application du projet SENSIB à d'autres milieux}

Dans le domaine marin, les pollutions ont toujours eu un impact fort sur les populations côtières. Un littoral souillé par un polluant engendre inexorablement une perturbation des écosystèmes et des risques pour les populations. Les pertes économiques pour la région touchée sont souvent importantes du fait des dommages subis par les secteurs du tourisme, de la pêche et des cultures marines. Dans ce contexte, le concept de sensibilité de l'environnement a été mis à profit notamment par l'IFREMER pour élaborer des atlas de vulnérabilité des zones côtières (IFREMER, 1997). L'extension de la méthodologie de l'IFREMER au cas des polluants radioactifs est l'un des enjeux du projet SENSIB pour les prochaines années (Duffa et al., 2007).

De même, dans le domaine des eaux continentales, les ressources en eau sont limitées en quantité et leur préservation est un enjeu essentiel car les activités humaines et la vie naturelle en sont largement tributaires. L'application du concept de sensibilité de l'environnement aux systèmes aquatiques continentaux est donc également envisagée dans le cadre du projet SENSIB (Eyrolle et al., 2005). 


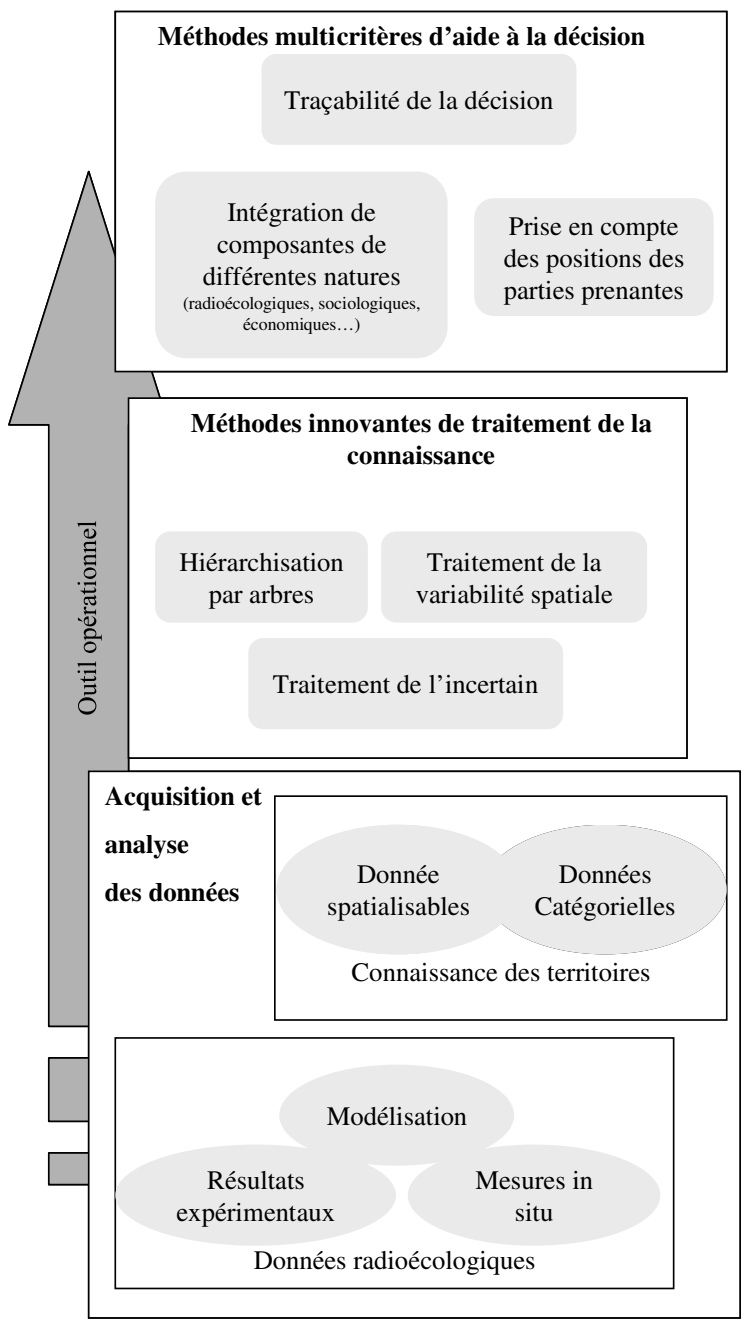

Figure 6 - Les données et méthodes fédérées par le projet SENSIB.

Data and methods gathered in the SENSIB project.

Enfin, le milieu atmosphérique est un milieu fondamental pour caractériser la réponse de l'environnement à une contamination radioactive car les conditions atmosphériques, en particulier les précipitations, ont un rôle prépondérant dans la répartition et l'intensité des dépôts. L'influence, sur l'efficacité de rabattement des polluants, des types de masses d'air et des formes de précipitations, i.e., brouillard, 
bruine, pluie, averse, neige, reste encore mal connue. L'amélioration de la connaissance de la répartition des radionucléides suite à des rejets atmosphériques mérite donc une classification des évènements climatiques et l'établissement d'une grille de variations des paramètres qui les caractérisent, en particulier du coefficient de lessivage et du flux de dépôt. C'est l'objectif poursuivi dans le cadre du volet atmosphérique du projet SENSIB.

\section{Conclusion}

La sensibilité radioécologique d'un territoire est définie comme l'aptitude des caractéristiques territoriales à amplifier, maintenir ou réduire les conséquences d'une pollution. L'objectif du projet Sensibilité Radioécologique est de définir des méthodes opérationnelles permettant de caractériser l'intensité de la réponse de l'environnement à une pollution radioactive. Le projet SENSIB s'appuie sur les connaissances radioécologiques acquises grâce aux observations de terrain, aux expérimentations en laboratoire et au développement de modèles ainsi que sur les données contextuelles, environnementales et anthropiques, propres aux territoires nationaux. En ce sens, SENSIB est un projet fédérateur. C'est aussi un projet innovant où sont déployées les avancées méthodologiques les plus récentes dans le domaine de l'analyse et du traitement des données. Les premiers résultats montrent que le concept de la sensibilité radioécologique permet de proposer des méthodes opérationnelles de caractérisation de l'état de l'environnement, notamment dans le contexte de la gestion post-accidentelle. Le projet SENSIB entre donc dans une nouvelle phase de transfert de la connaissance vers les utilisateurs potentiels, responsables et décideurs, et vers le public afin qu'à terme, les outils et les méthodes obtenus soient optimisés pour les besoins de la radioécologie opérationnelle.

Remerciements. Le projet SENSIB bénéficie du soutien financier de l'ADEME (convention $n^{\circ}$ 0472C0035).

\section{RÉFÉRENCES}

Aarkrog A. (1979) Environmental studies on radioecological sensitivity and variability with special emphasis on the fallout nuclides ${ }^{90} \mathrm{Sr}$ and ${ }^{137} \mathrm{Cs}$, In Ris-R-437, Ris National Laboratory (Denmark).

Anselin L. (1995) Local Indicators of Spatial Association - LISA, Geograph. Anal. 27, 93-115.

AVAIL (2002) Arctic Vulnerability to Radioactive Contamination, Final Report, Contract number IC15-CT98-0201, Borghuis A.M., Liland A., Strand P. (Eds), Norwegian Radiation Protection Authority.

Baudrit C. (2005) Représentation et propagation de connaissances imprécises et incertaines : application à l'évaluation des risques liés aux sites et aux sols pollués, Thèse soutenue le 19 octobre 2005, Université de Toulouse III (Informatique). 
Breiman L., Friedman J.H., Olshen R., Stone C.J. (1984) Classification and Regression Trees. Wadsworth, Belmont CA. U.S.A.

Briand B., Mercat-Rommens C. (2006) Difficulties and lessons of environmental data processing to fit modelling parameters, SETAC Europe 16th Annual Meeting, 7-11 May 2006, La Hague, The Netherlands.

Briand B., Mercat-Rommens C., Ducharme G. (2006) Apport de la biostatistique à la radioécologie de terrain, $38^{\mathrm{es}}$ journées de statistique, Clamart, 29 mai-2 juin 2006.

Brisson N., Gary C., Justes E., Mary B., Roche R., Ripoche D., Zimmer D., Sierra J., Bertuzzi P., Burger P., Bussière F., Cabidoche Y.M., Cellier P., Debaeke P., Gaudillère J.P., Maraux F., Seguin F.B., Sinoquet H. (2003) An overview of the crop model STICS, Eur. J. Agron. 18, 309332.

Chojnacki E., Ousny A. (1996) Description of the IPSN method for the uncertainty and sensitivity analysis and the associated software: SUNSET, in : ASME/JSME ICONE 4 Proceedings, Louisiana, USA. 3, 545-550.

Dempster A. (1967) Upper and Lower Probabilities Induced by a Multivalued Mapping, Ann. Math. Stat. 38, 325-339.

Descamps B., Guillet F. (2003) Enquête alimentaire dans trois secteurs de la Basse vallée du Rhône : Codolet, Tresques, Camargue. Consommation /Autoconsommation, Radioprotection 38, 299. 322.

Duffa C., Mercat-Rommens C., Thébault H. (2007) Radioecological sensitivity project on the French Mediterranean costal environment, $38^{\mathrm{e}}$ Congrès de la CIESM, Mediterranean Science Commission, Istanbul, Turquie, 9-13 avril 2007.

Durand V., Vray F., Mercat-Rommens C. (2006) Improving the knowledge of exposure by ingestion thanks to food surveys, International ISEE/ISEA Conference, Paris, 2-8 September 2006.

Durand V., Mercat-Rommens C., Curmi P., Benoit M., Briand B. (2007) Modelling regional impacts of radioactive pollution on permanent grassland, J. Agronomy (sous presse).

Eriksson A. (1997) Basic data for decisions on remediation of agricultural areas after a radioactive fallout, A report to the Swedish National Expert Group on Cleanup Swedish Radiation Protection Authority, Stockholm.

Eyrolle F., Louvat D., Métivier J.M., Rolland B. (2005) Origins and levels of artificial radionuclides within the Rhône river waters (France) for the last forty years: Towards an evaluation of the radioecological sensitivity of river systems, Radioprotection 40, 435-446.

Howard B.J., Wright S.M., Barnett C.L. (Eds.) (1999) Spatial analysis of vulnerable ecosystems in Europe: spatial and dynamic prediction of radiocesium fluxes into European foods (SAVE), Final report, 65 pp. Commission of the European Communities.

Howard B.J. et al. (2002) Radioecological Sensitivity Final Report, September 1998-March 2001, Center for Ecology and Hydrology, Natural Environment Research Council, March 2002.

IFREMER (1997) Atlas pour la préparation à la lutte et pour la lutte contre les pollutions marines accidentelles sur les côtes de Tunisie, application à la région de Sfax, Rapport de fin des phases I et II, avril 1997, Étude IFREMER, EMP, CEDRE, ENIS, REMPEC/IMO/UNEP.

INSEE (1991) Consommation et lieux d'achats des produits alimentaires en 1991, France, Institut national de la statistique et des études économiques.

Iosjpe M., Brown J., Strand P. (2002) Modified approach for box modelling of radiological consequences from releases into the marine environment, J. Environm. Radioact. 60, 91-103.

Le Ber F., Benoit M., Schott C., Mari J.-F., Mignolet C. (2006) Studying crop sequences with CarrotAge, a HMM-based data mining software, Ecol Model. 191, 170-185. 


\section{LA SENSIBILITÉ RADIOÉCOLOGIQUE DES TERRITOIRES}

Maystre L.Y., Pictet J., Simos J. (1994) Méthodes multicritères ELECTRE. Collection Gérer l'environnement, Presses Polytechniques et Universitaires Romandes (Lausanne, Suisse).

Mercat-Rommens C., Renaud P. (2005a) From field studies to risk management: the SENSIB Project, Radioprotection 40, S785-S790.

Mercat-Rommens C., Renaud P. (2005b) From radioecological sensibility to risk management: the SENSIB Project. 2nd International Conference on radioactivity in the environment, Nice, 2-6 October 2005.

Mercat-Rommens C., Chojnacki E., Merle-Szeremeta A., Brenot J., Sugier A. (2002) La nécessaire prise en compte des incertitudes dans les évaluations de risque : l'exemple du Groupe radioécologie Nord-Cotentin (GRNC), Environnement, Risques et Santé 5/6, 276-282.

Mercat-Rommens C., Chojnacki E., Baudrit C. (2005) Représentation et propagation de la connaissance imprécise: ce que les théories de l'incertain peuvent apporter aux sciences environnementales, $17^{\text {es }}$ journées scientifiques de la société d'écologie humaine, Arles, 23-25 novembre 2005.

Mercat-Rommens C., Chojnacki E., Baudrit C. (2006a) Ce que les théories de l'incertain peuvent apporter à l'évaluation des risques, Conférence $\lambda \mu 15$ « Risques et Performances », Lille, 10-12 octobre 2006

Mercat-Rommens C., Metivier J.M., Briand B., Durand V. (2006b) How geostatistics can help in predicting the level of radioactive contamination of cereals, 6th European Conference of Geostatistics for Environmental Applications, 25-27 October 2006, Rhodes, Grèce.

Moran P. (1950) Notes on continuous stochastic phenomena, Biometrika 37, 17-23.

Mourlon C., Calmon P. (2002) ASTRAL: a code for assessing situations after a nuclear accident, 12th annual meeting of SETAC Europe, Vienna, 12-16 May 2002, site Internet ASTRAL : https://www.irsn-astral.org.

Müller H., Pröhl G. (1993) ECOSYS-87: A dynamic model for assessing radiological consequences of nuclear accident, Health Phys. 64, 232-250.

Renaud P., Louvat D. (2004) Magnitude of fission product depositions from atmospheric nuclear weapon test fallout in France, Health Phys. 86, 353-358

Rommens C., Morin A, Merle-Szeremeta A. (1999) Le modèle FOCON d'évaluation de l'impact dosimétrique des rejets radioactifs atmosphériques des installations nucléaires en fonctionnement normal, Radioprotection 34, 195-209.

Strand P., Balonov M., Bewers M., Howard B.J., Tsaturov Y., Salo A. et al. (Eds.) (1997) Arctic Monitoring and Assessment Program. AMAP, Norwegian Radiation Protection Authority (Oslo) 